\title{
Uso de estrategias metacognitivas para la comprensión textual*
}

\author{
ÁNGELA ELVINIA MUÑOZ MUÑOZ* \\ Fundación Universitaria Juan de Castellanos, Colombia \\ amunoz@jdc.edu.co
}

MYRIAM OCAÑA DE CASTRO**

Docente (r) Institución Educativa José Acevedo y Gómez, Colombia merchasmo4@yahoo.com

Recepción: 04 de marzo de 2016

Aprobación: 23 de mayo de 2016

Forma de citar este artículo: Muñoz-Muñoz, Á.E, \& Ocaña de Castro, M. (2017). Uso de estrategias metacognitivas para la comprensión textual. Cuadernos de Lingüística Hispánica, (29), 223-244. doi: http://dx.doi.org/10.19053/0121053X.n29.2017.5865

\footnotetext{
* Artículo de investigación.

** Magíster en Lingüística y Directora del Departamento de Humanidades Fundación Universitaria Juan de Castellanos. Código ORCID: orcid.org/0000-0001-7029-7223

*** Magíster en Lingüística. Docente tutora programa "Todos aprenden" Monguí, Boyacá, Colombia. Código ORCID: orcid.org/00000002-0100-0622
} 


\section{Resumen}

Este artículo presenta resultados de una investigación cuyo objetivo principal se centró en orientar la implementación de estrategias metacognitivas para mejorar la comprensión lectora y, en especial, de inferencias en textos expositivos, mediante una intervención pedagógica apoyada en tres procesos: antes, durante y después de la lectura. La metodología utilizada fue de tipo pre-experimental, con un diseño pre test- post test, sin grupo de control. Según la comparación de los promedios con la $t$-Student para muestras dependientes, con una significación del 0,05 , se concluyó que el uso de las estrategias metacognitivas influyó favorablemente en el proceso lector de estudiantes de secundaria, Grado Octavo, de dos Instituciones Educativas públicas de los municipios de Nuevo Colón y Samacá (Boyacá, Colombia).

Palabras clave: cognición, metacognición, comprensión de lectura, estrategias metacognitivas, inferencias.

\section{The Use of Metacognitive Strategies to Enhance Reading Comprehension Skills}

\section{Abstract}

This article presents the outcome of a research project whose main objective was to foster the implementation of metacognitive strategies, designed to enhance reading comprehension skills, especially regarding the inferential level of reading in expository texts. The pedagogical intervention for this research is supported in three fundamental moments: before, during and after reading. A pre-experimental methodology, with a pre test/post test design, and without a control group, was implemented for this project. According to a comparison of average scores using the Students' t-Test for independent samples, a 0,05 level of significance was found, proving that the use of metacognitive strategies had a positive influence in the reading process of high school students of the eighth grade in two public educational institutions from the municipalities of Nuevo Colón and Samacá (Boyacá, Colombia).

Key words: Cognition, Metacognition, Reading Comprehension, Metacognitive Strategies, Inferences. 


\section{Utilisation des stratégies métacognitives pour la compréhension textuelle}

\section{Résumé}

Cet article présent des résultats d'une recherche dont l'objectif principal s'est centré sur le fait d'orienter la mise en œuvre des stratégies métacognitives. Pour améliorer la compréhension de lecture et, en particulier, d'inférences des textes expositifs, à travers une intervention pédagogique appuyée sur trois processus: avant, pendant et après la lecture. La méthodologie utilisée a été de type expérimental, avec un design pré-test, posttest, sans groupe de contrôle. Selon la comparaison des moyennes avec la $t$-Student pour des échantillons dépendants, avec une signification de 0,05 , on a conclu que l'utilisation des stratégies métacognitives a eu une influence favorable sur le processus de lecture des élèves de l'école secondaire, Classe de Troisième, de deux institutions Éducative des communes de Nuevo Colón et Samacá (Boyacá, Colombie).

Mots clés: cognition, métacognition, compréhension de lecture, stratégies métacognitives, inférences.

\section{Uso de estratégias metacognitivas para a compreensão textual}

\section{Resumo}

Este artigo apresenta resultados de uma pesquisa cujo objetivo principal centrou-se em orientar a implementação de estratégias metacognitivas para melhorar a compreensão leitora e, em especial, de inferências em textos expositivos, mediante uma intervenção pedagógica apoiada em três processos: antes, durante e depois da leitura. A metodologia utilizada foi do tipo pré-experimental, com um desenho pre test- post test, sem grupo de controle. Segundo a comparação das médias com a $t$-Student para amostras dependentes, com uma significação de 0,05 , concluiu-se que 0 uso das estratégias metacognitivas influiu favoravelmente no processo leitor de estudantes de secundária, do $9^{\circ}$ ano, de duas Instituições Educativas públicas dos municípios de Nuevo Colón e Samacá (Boyacá, Colômbia).

Palavras chave: cognição, metacognição, compreensão de leitura, estratégias metacognitivas, inferências. 


\section{Introducción}

Desde la década de los sesenta, hasta la actualidad, existen evidencias que corroboran que los estudiantes de básica secundaria no desarrollan habilidades de comprensión de lectura y por consiguiente no realizan deducciones e inferencias, que les permitan aplicar a su vida diaria, los principios y valores que se transmiten a través de la lectura; por lo tanto, las estrategias utilizadas hasta el presente se deben revaluar y proponer otras de tipo pedagógico que superen la idea de que la lectura es una actividad pasiva de decodificación y reconocer que es una actividad que involucra variados y complejos procesos intelectuales; en consecuencia, esta investigación propone la implementación, dentro de las aulas de educación básica secundaria colombianas, de estrategias metacognitivas antes, durante y después de la lectura, dirigidas a remediar las carencias de las habilidades mencionadas anteriormente, para lograr que la lectura no solo sea placentera, sino útil y significativa.

\section{Sustentación Teórica}

La presente investigación tiene como bases teóricas la cognición, la metacognición, la lingüística, la psicolingüística, la textolingüística y la didáctica de la comprensión de lectura. En cuanto a la cognición y la metacognición, se toma como referente a Flavell (1979), quien utilizó el término de metamemoria, término al cual acompañaron otros dos vocablos vinculados con él: metacognición y metacomprensión. Con el fin de analizar el conocimiento acerca de la cognición, Flavell y Wellman (1977) plantean que el uso de los recursos cognitivos propios no es espontáneo sino que cuando se tiene la necesidad de enfrentar tareas o problemas concretos, es prioridad activarlo, a fin de seleccionar la estrategia más pertinente en cada situación. Desde esta perspectiva, Brown, Armbruster, \& Baker (1986) confirman lo anterior, ya que definen la metacognición como "el control deliberado y consciente de la acciones cognitivas" (p. 453). Las actividades metacognitivas son los mecanismos autorregulatorios que utiliza un sujeto durante la resolución de un problema 0 al enfrentarse a una tarea. Con respecto al proceso de estrategias metacognitivas en la lectura, esta investigación siguió el modelo de Ríos (1991), quien define "la metacomprensión como el conocimiento acerca de los recursos cognitivos para enfrentar una tarea de lectura y la autorregulación que ejerce sobre las estrategias como lector" (p. 278). La actividad de autorregulación en la lectura, según este autor, comprende tres fases, a saber: planificación, supervisión y evaluación. 
En la actualidad, investigaciones como las de Chirilos (2014) definen las estrategias metacognitivas en el proceso de investigación científica, por ejemplo, Sanz (2010) concibe la metacognición como "la actividad mental centrada en el propio funcionamiento psicológico; es conciencia y regulación del mundo interior, en oposición a las actividades centradas en la información proporcionada por el mundo exterior" (p. 111); además, describe la autorregulación, mediante las etapas de planificación (antes), monitorización (durante) y evaluación (después), etapas que son objeto de estudio en la presente investigación y que en su momento las sustentaron Ríos (1991) y Cooper (1998), quienes identifican los procesos mentales, las actividades cognitivas, las fases del proceso lector (pre-lectura, durante la lectura y post-lectura), las estrategias metacognitivas y las actividades metacognitivas.

Por otra parte, desde la perspectiva del modelo interactivo, Castillo y Galvis (1997) asumen la comprensión lectora como un proceso constructivo en el que se requiere el esfuerzo deliberado del lector para ir interpretando el significado de un texto; en este modelo intervienen tres componentes: el sujeto, el texto y el contexto. Los tres componentes establecen un proceso dialéctico, cada uno aporta elementos al proceso de comprensión lectora. Conviene distinguir entonces, los componentes que aporta cada uno de los tres elementos en el proceso de comprensión que a continuación se describen:

El texto, ofrece informaciones de tipo lingüístico, textual y discursivo (1977). Las informaciones de tipo lingüístico se presentan a nivel intra-oracional; estas informaciones corresponden al sistema formal de la lengua (gramática). La información de tipo textual es inter-oracional, es decir,

La manera como se integran las oraciones en el nivel textual, conforman una unidad semántica constituida por dos planos, el del contenido y el de la expresión (coherencia y cohesión); y por último, la información discursiva (pragmática) tiene que ver con la situación comunicativa, el tipo de discurso y las relaciones entre el texto y el contexto. (Tardif, 1997, p. 183).

El contexto, se relaciona con los elementos del entorno en el cual se lleva a cabo el proceso lector; así, el contexto de acuerdo con los autores Santiago, Castillo y Vega (2005),

corresponde a todas aquellas situaciones que rodean y propician la interacción entre el texto y el lector, de tal manera que aparecen multiplicidad de factores alrededor del acto lector: propósitos del lector, tipo de texto, condiciones de lectura, situación específica, entre otros. (p. 23).

El lector, aporta todos sus conocimientos, procesos mentales e intereses. El lector se constituye en elemento esencial, ya que es él quien posibilita la reconstrucción del significado 
del texto, a partir de la interacción entre sus conocimientos previos, la información que ofrece el texto y el contexto.

En la interacción texto-lector, esta aporta tres tipos de conocimientos: el primero, que son los declarativos, corresponden al qué, están asociados al conocimiento del mundo en general, la lengua y la escritura; los conocimientos procedimentales se relacionan con el cómo el lector adelanta el proceso lector, es decir, tienen relación con los procesos y estrategias de orden cognitivo que permiten enlazar la información previa del lector con la información que proporciona el texto para su comprensión (Tardif, 1997). El tercer conocimiento pertenece a los condicionales que tienen que ver con la acción y están asociados al cuándo y al porqué adelantar una tarea; Tardif (1997) afirma que el lector debe "recurrir a la estrategia adecuada en el momento justo" (p. 187), de tal forma que le permita al lector seleccionar las estrategias, los recursos y los procedimientos necesarios para realizar la lectura. Dentro de estos conocimientos condicionales se incluyen las estrategias metacognitivas que le posibilitan al estudiante controlar la realización de la tarea, en la medida en que define los objetivos, establece estrategias, supervisa el proceso y evalúa el resultado.

Lo que viene ahora es identificar los tipos de contenidos que debe enseñar el maestro a sus estudiantes. Con respecto a este tema, Rincón (2003) dice que el papel del docente en el proceso de la comprensión textual apunta a ofrecer al estudiante el andamiaje necesario que lo lleve a ser lector autónomo.

Los resultados de las investigaciones recientes como la de Patiño y Restrepo (2013) realizada con niños de los grados primero y quinto primaria; la de Heit (2011) con adolescentes; la de Chirilos (2014) con estudiantes de posgrado y la de Muñoz y Ocaña (2008) desarrollada con adolescentes, evidencian los resultados positivos del entrenamiento en estrategias metacognitivas, para el mejoramiento del aprendizaje y la comprensión de lectura.

En nuestro estudio, se enfatizó en que los estudiantes utilizaran los procesos cognitivos en forma estratégica; por lo tanto, se les enseñó cuáles son esos procesos cognitivos (memoria, atención y percepción), para qué sirven, cuándo utilizarlos y el porqué utilizarlos, en función de los otros procesos cognitivos superiores (inferenciales y estratégicos).

El aprendizaje significativo que tiene como base la teoría constructivista afirma que el aprendizaje del estudiante parte de sus pre-conceptos y los constantes equilibrios y desequilibrios intelectuales; es decir, se apoya en lo que la Psicolingüística denomina el concepto de mundo, que solamente se modifica a través del aprendizaje proporcionado 
por la interacción entre el lector y el texto, esto conlleva la elaboración de un conocimiento propio, y si se ponen en práctica estos aprendizajes en otros estudiantes, se espera formar alumnos más autónomos, reguladores de sus propios procesos de pensamiento, que conocen, emplean y aplican en diversas situaciones y en forma más provechosa las estrategias metacognitivas.

El diseño de la presente investigación comprende el desarrollo de secuencias didácticas que enseñan y ponen en práctica determinadas estrategias metacognitivas aplicadas durante el proceso lector mediante tres fases: antes, durante y después de la lectura, para así garantizar un proceso lector consciente y dinámico; por lo tanto, el estudio aporta una herramienta de enseñanza para profesores de secundaria y de educación superior interesados en mejorar la comprensión lectora de sus estudiantes, garantizar el aprendizaje y propiciar en ellos habilidades comunicativas, argumentativas y personales que sean aplicables, no solo a la lectura, sino a cualquier situación de la vida cotidiana.

En cuanto a la tipología textual, la investigación se basa en la Textolingüística que da unos principios organizadores al discurso y su jerarquización. El texto que se analiza en esta investigación es de tipo expositivo-explicativo, es decir, da información, aporta conocimientos, maneja vocabulario especializado y transmite saberes, a diferencia del texto narrativo de ficción, que contribuye a alimentar la imaginación a través de personajes, tiempo, espacio, narradores, etc.

El texto expositivo habla de cuestiones reales (no imaginarias), las que el lector debe ir contrastando con sus conocimientos previos a lo largo de la lectura; también permite hacer inferencias en torno de la información que presenta. Al respecto, Álvarez (1996) afirma:

La superestructura o principios organizadores de los textos expositivos no obedecen a una superestructura común, como en el narrativo, sino que se ajusta a la siguiente organización: definición-descripción, clasificación-colección, comparación y contraste, problema-solución, pregunta-respuesta y causa-consecuencia. (p. 35).

En síntesis, la cognición, la metacognición, la Textolingüística y la Psicolingüística son esenciales en las investigaciones actuales sobre procesos de pensamiento superior y procesos de interacción entre el lector y el texto. En palabras de Lomas (1999), hasta ahora "la investigación sobre la lectura se ha encaminado fundamentalmente a la descripción pormenorizada de lo que el lector tiene que saber y saber hacer para leer un texto" (p. 328), temática que es expuesta en la presente investigación. 
Por lo anterior, se formuló esta pregunta de investigación: ¿Cómo implementar en estudiantes de básica secundaria, estrategias pedagógicas de tipo cognitivo y metacognitivo que mejoren la comprensión lectora de textos expositivos, la construcción de pensamiento significativo y la autorregulación en procesos inferenciales?

\section{Hipótesis}

La siguiente fue la hipótesis que orientó el presente trabajo: si se aplican estrategias basadas en la cognición y la metacognición en la lectura de textos expositivos, se mejorará significativamente la comprensión de este tipo de textos.

\section{Metodología}

Esta investigación de tipo pre-experimental se desarrolló con un diseño pre test, post test y sin grupo de control. La población intervenida fueron 94 estudiantes de Grado Octavo (todos con edades comprendidas entre 12 y 14 años): 30 del Colegio Salamanca de Samacá y 64 del Colegio Nuestra Señora La Antigua de Nuevo Colón. Se les aplicó una prueba pre-test sobre el uso de estrategias metacognitivas; luego se realizó el proceso didáctico consistente en enseñarles el manejo de las mismas; una vez asimiladas, se les aplicó el pos-test, con el fin de comprobar si hubo mejoramiento en el uso y manejo de dichas estrategias y en la comprensión de textos expositivos.

La operacionalización de la variable independiente se hizo a través de las fases de planificación, supervisión y evaluación. La fase de planificación comprendió las siguientes estrategias: conocimientos previos, objetivos de la lectura, plan de acción, conocimiento sobre la superestructura y macroestructura de los textos expositivos, aproximación 0 alejamiento de la meta, detección de aspectos importantes (vocabulario e información relevante del texto), detección de conocimientos sobre el texto, reconocimiento de dificultades en la comprensión, conocimiento de las causas de las dificultades, flexibilidad en el uso de las estrategias y evaluación de la efectividad de las estrategias usadas.

La operacionalización de la variable dependiente, es decir, la habilidad de la comprensión lectora fue detectada mediante el manejo de la comprensión de vocabulario, comprensión de detalles, comprensión para descubrir la macroestructura del texto, comprensión para la identificación de la idea principal y la comprensión de inferencias: léxicas, de causalidad, de comparación, de especificación, de inclusión y macroestructurales. El test, instrumento de la investigación, con el cual se detectó la variable dependiente se construyó con técnicas psicométricas, según las siguientes etapas: inicialmente se especificaron las estrategias metacognitivas, luego se definieron los criterios de comprensión de lectura y, por último se tuvieron en cuenta los criterios de inferencia. Estos tres aspectos: 
estrategias, criterios de comprensión y de inferencia se precisaron, según las teorías de la Psicolingüística y la Textolingüística. Con base en los anteriores criterios, se construyó una tabla de especificaciones, y en ella se fijó el número y tipo de preguntas, tanto para las estrategias metacognitivas, como para la comprensión y las inferencias. De acuerdo con lo anterior, se puede argumentar psicométricamente que el test posee una confiabilidad de consistencia interna y una validez de tipo aparente, como una validez de constructo.

A continuación se presenta el test, de las estrategias metacognitivas, con la estructura completa, solamente con los dos primeros ítems de cada categoría, debido a su extensión (7 páginas):

\section{Tabla 1. Test para detectar el uso de estrategias metacognitivas}

TEST O PRUEBA PARA DETECTAR SI EL ESTUDIANTE UTILIZA LAS ESTRATEGIAS
METACOGNITIVAS
Instrucciones:
- Número de identificación: (a los estudiantes se les da un número para Identificar la cantidad de pruebas
aplicadas; pero el test se responde en forma anónima).
- Estudiante: por favor responda la siguiente prueba en forma anónima, es decir, no escriba algo que le
permita ser identificado personalmente.
- La prueba siguiente no tiene connotaciones de bueno o malo, ni connotaciones de calificación
- Readémica.
- Señanale con una X en la casilla de SÍ o NO según sea su conocimiento.

\begin{tabular}{|c|c|c|c|}
\hline CATEGORÍAS & $\begin{array}{c}\text { PREGUNTAS PARA ACTIVAR LA AUTORREGULACIÓN } \\
\text { Y TOMA DE CONCIENCIA }\end{array}$ & \multirow[b]{2}{*}{ Sí } & \multirow[b]{2}{*}{ NO } \\
\hline \multicolumn{2}{|r|}{ FASE DE PLANIFICACIÓN } & & \\
\hline $\begin{array}{l}\text { Conocimientos } \\
\text { Previos }\end{array}$ & $\begin{array}{l}\text { - Al comenzar a leer, me pregunté: ¿Qué sabía sobre el tema de la } \\
\text { lectura? } \\
\text { - ¿Me formulé preguntas sobre el tema del texto? }\end{array}$ & & \\
\hline Objetivos de la lectura & $\begin{array}{l}\text { - Con ayuda del profesor tracé los objetivos de la lectura. } \\
\text { - Tracé de forma autónoma los objetivos de la lectura. }\end{array}$ & & \\
\hline Plan de acción & $\begin{array}{l}\text { - ¿Realicé y seguí un plan de acción para efectuar la lectura? } \\
\text { - Revisé el texto: extensión, tamaño de la letra, tablas, diagramas, } \\
\text { ilustraciones. }\end{array}$ & & \\
\hline $\begin{array}{l}\text { Conocimiento sobre } \\
\text { la superestructura y } \\
\text { la macroestructura de } \\
\text { los textos expositivos }\end{array}$ & $\begin{array}{l}\text { - ¿Hice uso de estrategias para construir el contenido textual? } \\
\text { - ¿Hice un listado para seleccionar la información que me pareció a } \\
\text { mí importante? } \\
\text { - Macroestructura: } \\
\text { ～Releí el texto antes de inferir las ideas fundamentales. }\end{array}$ & & \\
\hline
\end{tabular}




\begin{tabular}{|c|c|c|c|}
\hline CATEGORÍAS & $\begin{array}{c}\text { PREGUNTAS PARA ACTIVAR LA AUTORREGULACIÓN } \\
\text { Y TOMA DE CONCIENCIA }\end{array}$ & & \\
\hline \multicolumn{2}{|r|}{ FASE DE SUPERVISIÓN } & Sí & No \\
\hline $\begin{array}{l}\text { Aproximación o } \\
\text { alejamiento de la } \\
\text { meta }\end{array}$ & $\begin{array}{l}\text { - ¿Revisé si estaba logrando los objetivos que me propuse con la } \\
\text { lectura? ¿Relacioné lo que iba leyendo con mis propósitos de } \\
\text { lectura? }\end{array}$ & & \\
\hline $\begin{array}{l}\text { Detección de aspectos } \\
\text { importantes }\end{array}$ & $\begin{array}{l}\text { - Vocabulario: } \\
\text { ～Recurrí a las palabras conocidas en unas frases o en un párrafo } \\
\text { para determinar el significado de una palabra desconocida. } \\
\text { - Determinar información relevante del texto: } \\
\text { ～Identifiqué la idea general que el autor determinó en el texto. } \\
\text { ～Identifiqué los detalles que el autor utilizó para sustentar la idea } \\
\text { central. }\end{array}$ & & \\
\hline $\begin{array}{l}\text { Detección de } \\
\text { conocimientos sobre } \\
\text { el texto }\end{array}$ & $\begin{array}{l}\text { - ¿Se me facilitó la comprensión del texto porque tenía los } \\
\text { conocimientos previos? } \\
\text { - Distinguí claramente entre lo que ya sabía y la información nueva. }\end{array}$ & & \\
\hline $\begin{array}{l}\text { Detección de } \\
\text { dificultades en la } \\
\text { comprensión }\end{array}$ & $\begin{array}{l}\text { - ¿Me di cuenta de que tenía dificultades de comprensión porque no } \\
\text { tenía los conocimientos previos necesarios? } \\
\text { - Determiné cuáles fueron las partes del texto más difíciles de } \\
\text { comprender. }\end{array}$ & & \\
\hline $\begin{array}{l}\text { Conocimiento de } \\
\text { las causas de las } \\
\text { dificultades }\end{array}$ & $\begin{array}{l}\text { - Me di cuenta que no tenía conocimientos previos adecuados sobre } \\
\text { el tema. } \\
\text { - Pensé que la lectura iba a ser muy compleja. }\end{array}$ & & \\
\hline $\begin{array}{l}\text { Flexibilidad en el uso } \\
\text { de las estrategias }\end{array}$ & $\begin{array}{l}\text { - Para identificar mis conocimientos previos, me basé en el título } \\
\text { del texto. } \\
\text { - Cuando me di cuenta de que no estaba comprendiendo el texto, } \\
\text { acudí a estrategias de comprensión que conocía previamente. }\end{array}$ & & \\
\hline \multicolumn{2}{|r|}{ FASE DE EVALUACIÓN } & Sí & NO \\
\hline $\begin{array}{l}\text { Evaluación de la } \\
\text { efectividad de las } \\
\text { estrategias usadas }\end{array}$ & $\begin{array}{l}\text { - ¿Qué pasos llevados a cabo durante la lectura me facilitaron la } \\
\text { comprensión del texto? } \\
\text { - ¿Evalué mi comprensión del texto a través de la comparación con } \\
\text { mis compañeros? }\end{array}$ & & \\
\hline
\end{tabular}


En la siguiente tabla se identifican los aspectos de comprensión de lectura y de inferencias:

Tabla 2. Comprensión de lectura y de inferencias

\begin{tabular}{|l|l|}
\hline ASPECTOS DE LA COMPRENSIÓN DE LECTURA & ASPECTOS DE LA COMPRENSIÓN DE INFERENCIAS \\
\hline - Comprensión de vocabulario. & - Inferencias léxicas. \\
- Comprensión de detalles. & - Inferencias de causalidad. \\
- Comprensión para realizar la macroestructura & • Inferencias de comparación. \\
$\begin{array}{l}\text { del texto. } \\
\text { - Comprensión para la identificación de la idea } \\
\text { principal. }\end{array}$ & - Inferencias de especificación. \\
\hline
\end{tabular}

El entrenamiento consistió en el desarrollo de una secuencia de doce (12) sesiones que comprendieron talleres o didácticas para el conocimiento y aplicación de estrategias metacognitivas de comprensión de lectura e inferencias. Cada una de las sesiones se realizó con la siguiente estructura: Tiempo: dos o cuatro horas; Tema: según la fase y estrategia metacognitiva utilizada; sustento teórico; objetivos y metodología por parte del profesor: se da a conocer el plan de acción, los estudiantes consignan en su cuaderno el plan de acción; Motivación al estudiante: actividades dirigidas para estimular la participación del estudiante y favorecer su aprendizaje, además, para que sepa que tiene un potencial cognitivo y que mediante este puede elaborar procesos de pensamiento; Enseñanza explícita: en ella se dan criterios para la aplicación de las estrategias metacognitivas; creación de conciencia de los procesos que se requieren; el desarrollo de la sesión: en ella se desarrollan las actividades específicas propuestas, de acuerdo con los temas de la sesión; la última fase va dirigida al estudiante, mediante la Autoevaluación: preguntas autorreguladoras del proceso abordado en la sesión; verificación de logros alcanzados: reflexión personal y grupal sobre el cumplimiento de los objetivos señalados; Aspectos por mejorar: detección de dificultades durante la sesión y propuesta de acciones correctivas. Ahora se describe la sesión 1 con la estructura completa, pero sin el contenido en su totalidad, debido a la extensión. 
Tabla 3. Fase 1. Antes de la lectura

\begin{tabular}{|l|l|}
\hline \multicolumn{2}{|c|}{ FASE 1: ESTRATEGIAS METACOGNITIVAS ANTES DE LA LECTURA } \\
\hline SESIÓN: $\mathrm{N}^{\circ} .1$ & DURACIÓN: 2 HORAS \\
\hline TEMA: Conocimientos Previos & SUSTENTO TEÓRIC0: \\
La información previa ha sido definida como "la sumatoria de los aprendizajes previos y el \\
desarrollo anterior de un individuo; en una palabra, la experiencia [...] El desarrollo de la \\
información previa ha de desarrollarse con el tema específico y los conceptos del texto. La \\
evaluación de la información previa de que disponen los alumnos se efectúa a través de la \\
observación y el conocimiento de los niños." (Cooper, 1998, p. 111).
\end{tabular}

\section{OBJETIVOS DE LA SESIÓN:}

Desarrollar la habilidad de activar conocimientos previos antes de iniciar cualquier lectura:

- Ser consciente de que la información previa ayuda a comprender la lectura. Preguntarse: ¿qué sabe sobre el tema de lectura?, ¿qué estrategias utilizó para activar los conocimientos previos?, ¿el texto proporciona ilustraciones, tablas, fotografías, palabras claves o resaltadas, que le ayudan a recordar algo de la lectura?

- Ser consciente de la necesidad de planificar una actividad, para tener éxito en ella.

- Ser consciente de que la información que se tiene sobre un tema, se integra con la nueva para convertirse en un nuevo esquema, construcción de pensamiento, o nuevo aprendizaje.

- Ser consciente que mediante el uso de material auditivo y visual, se aprende fácilmente.

\section{METODOLOGÍA DE LA SESIÓN}

\section{～Profesor:}

El docente da a conocer el plan de acción que se va a ejecutar. Los estudiantes consignan en el cuaderno el plan de acción.

- Ejercicio de atención.

- Presentación de los objetivos de la sesión.

- Desarrollo de conocimientos previos, mediante la socialización de los estudiantes del tema del texto.

- Presentación de un video sobre el texto.

Motivación: dirigida a que el estudiante sepa que tiene un potencial cognitivo, y que mediante este, puede elaborar procesos de pensamiento. La actividad de motivación se orienta a desarrollar un ejercicio de atención, percepción y memoria espacial. 


\section{Enseñanza explícita: encaminada a:}

- Aplicar la estrategia cognitiva de examinar, es decir, dar un vistazo al material asignado, para formar un marco mental o esquema.

- Tener criterio del porqué utilizar estrategias metacognitivas.

- Acceder al conocimiento previo. Pensar (recordar) lo que se conoce sobre el tema.

\section{Desarrollo de la sesión:}

Se le dice a los estudiantes que van a leer un texto titulado "Un viaje musical por el mundo", antes de hacer la lectura, se hará una reflexión de algunas ideas importantes de la lectura.

- Plan de acción antes de la lectura.

- Conciencia de la utilización de estrategias metacognitivas.

〜 Profesor - estudiantes: encaminada a la enseñanza del conocimiento procesual (conocimiento del cómo o proceso); conocimiento condicional (conocimiento del cuándo y por qué).

Estudiante. encaminada a la enseñanza de la autoevaluación en el cumplimiento de la tarea, del aprendizaje de las estrategias metacognitivas, de su aplicación en la lectura para demostrar comprensión mediante la transferencia del conocimiento aprendido a una situación similar, pero nueva; reconocer, identificar o admitir un problema en el caso de que no se cuente con conocimientos previos sobre el tema.

- Autoevaluación: el estudiante se autoevaluará sobre su participación y cumplimiento del plan de acción, respondiendo a los siguientes interrogantes: ¿realizó y siguió un plan de acción para efectuar la lectura?; ¿a partir del título de la lectura identificó de qué se trataba?; irevisó el texto: su extensión, tamaño de la letra, ilustraciones y tablas?

- Verificación de los logros alcanzados: analizar la efectividad de la estrategia, comprender los procesos cognitivos y metacognitivos implicados en el uso de la estrategia. Hacer énfasis en la importancia del aprendizaje, a través de los sentidos auditivo y visual.

- Aspectos por mejorar: evaluar el avance en la solución del problema, es decir, si adquirió o no conocimientos nuevos. 


\section{Análisis y discusión de resultados}

A continuación aparece el análisis y la interpretación de los resultados del Pre-Test y del Post-Test:

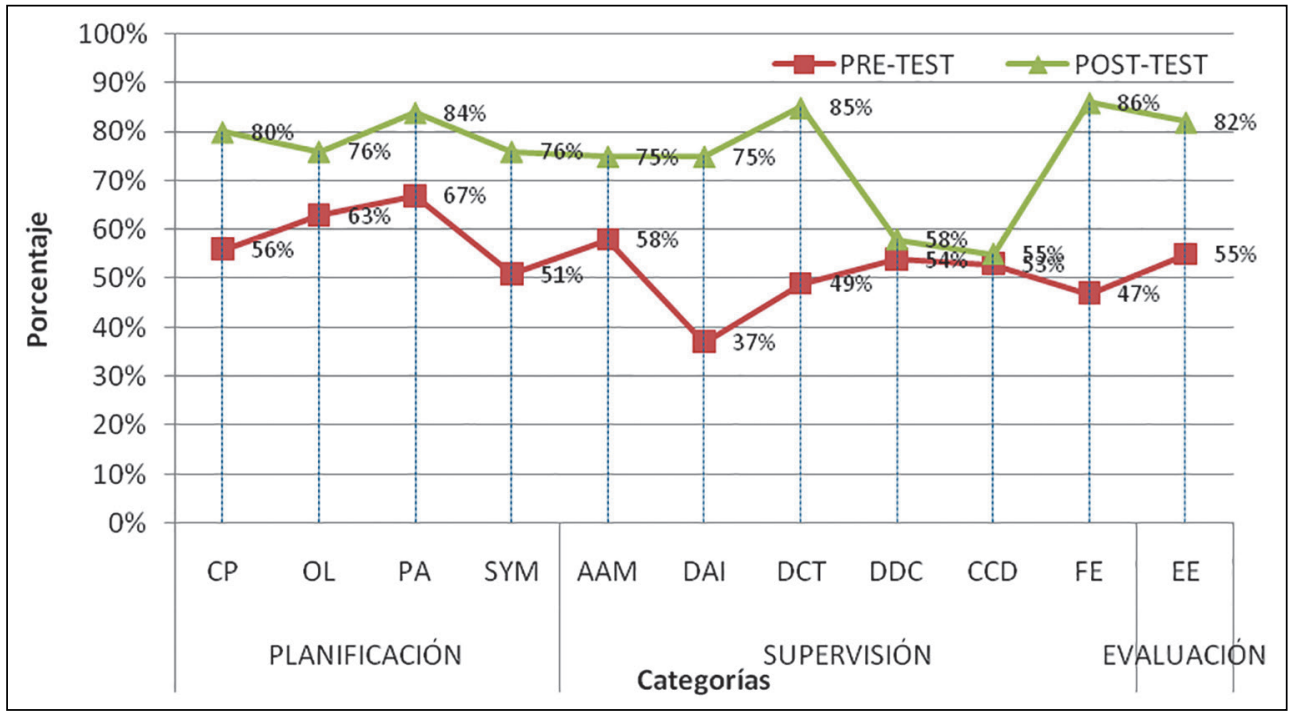

Figura 1. Comparación de los porcentajes de respuestas de estrategias metacognitivas utilizadas en el Pre-Test y Post-Test

A partir de los resultados obtenidos, según los valores $t$-Student con una significación del 0.05, el uso de las Estrategias Metacognitivas influyó directamente en mejorar la comprensión del texto e inferencias en los estudiantes. Para sustentar lo anterior, se analizaron los resultados según cada estrategia metacognitiva y su efecto en la comprensión de acuerdo con las cifras obtenidas en el estudio. A continuación se describen algunos resultados de esta primera fase:

Si se observa la figura 1 , los resultados del post-test en relación con el pre-test son superiores, lo cual indica un incremento después de la intervención. En términos generales, en la fase de planificación, se comprueba que, por una parte, los estudiantes lograron adquirir la habilidad en el uso de las estrategias metacognitivas para tal fin y, por otra, que los estudiantes utilizan en mayor medida como estrategias de planificación: plan de acción y conocimientos previos en el proceso de la lectura, dada su utilidad y efectividad en la comprensión del texto e inferencias. Es de anotar que desde el modelo interactivo, el lector aporta sus conocimientos previos, de ahí que Solé (1994) considere que "antes de la lectura 
se debe ayudar al estudiante en el proceso de comprensión a través de estrategias" (p. 89), una de ellas es revisar y actualizar el conocimiento previo; al respecto, Cooper (1998) afirma que la información previa es un componente fundamental en todo programa de comprensión antes de leer cualquier texto.

Por otro lado, el entrenamiento de los estudiantes en la estrategia de Objetivos de la Lectura permitió que después de la intervención, presentaran más destreza en plantear los objetivos al realizar la lectura. El porcentaje inicial de 63\% aumentó al $76 \%$ en el posttest, cifras significativas. Por consiguiente, puede afirmarse que los estudiantes aprendieron a formular y seguir objetivos antes de realizar la lectura.

Además, la estrategia Plan de Acción que hace parte de la fase de planificación, como lo señala Ríos (1991), consiste en hacer una lectura general y cuestionarse si se realiza o no un plan de acción para efectuarla, lo que implica revisar el texto, analizar los párrafos e identificar el título y el tema de la lectura. En este estudio, el desempeño de los estudiantes en el uso de la estrategia mejora en un 17\%: del pre-test $67 \%$ al post-test $84 \%$, diferencia significativa con el $95 \%$ de confianza, esto quiere decir, que el tratamiento experimental tuvo un efecto en la elaboración de planes de acción en la lectura por parte de los estudiantes.

Respecto de la estrategia superestructura y macroestructura de texto, que se evidencia cuando el estudiante identifica el tema, las ideas secundarias, la relación entre las ideas fundamentales, la información textual y los conocimientos previos, las cifras obtenidas indican un aumento en el porcentaje de respuestas positivas, pasando del 51\% al $76 \%$; aquí, se denota una diferencia del $15 \%$ respecto del porcentaje inicial; el valor $t$-student es significativo al 0.05 , lo cual comprueba que: 1) los estudiantes aprendieron a utilizar esta estrategia y 2) que resulta útil para mejorar la comprensión del texto, especialmente la comprensión de la superestructura y macroestructura, en donde se presentó un aumento significativo.

Como segunda fase se planteó la Supervisión, estos son algunos aspectos importantes: los estudiantes, a través del programa de intervención, aprendieron a supervisar el plan de acción trazado, a modificarlo y ajustarlo de acuerdo con sus características personales, mostrando cada vez más autonomía y capacidad reflexiva; luego, cada estudiante diseñó su propio plan, inicialmente dirigido y planteado por el docente y, finalmente, liderado por ellos mismos como se comprobó en el estudio.

Acerca de la estrategia detección de aspectos importantes, una de las más útiles en la comprensión de lectura, se observó un alto incremento en el porcentaje que los estudiantes alcanzaron en el post-test, inicialmente se obtuvo el $37 \%$ y después el 
$75 \%$ consiguiendo una diferencia del $38 \%$ en su desempeño, proporciones significativas al 0.05 , resultados que evidencian la adquisición de los estudiantes de la habilidad para desarrollar la estrategia y la preferencia por el uso de la misma; así como la incidencia en la comprensión de la idea principal, detalles y vocabulario primordialmente.

En la intervención se enfatizó en la importancia de no pasar por inadvertida ninguna palabra desconocida, pues es necesaria la búsqueda de su significado, ya sea en el diccionario, en la asociación con conocimientos previos, la consulta a expertos o como lo plantea Solé (1994), una de las habilidades de lectura que es necesario incentivar es el reconocimiento del significado de las palabras a partir del contexto.

Por otro lado, en la estrategia detección de conocimientos sobre el texto, se observó un incremento importante del 36\%, en el pre-test se obtuvo el $49 \%$ mientras en el post-test. el $85 \%$, siendo significativos al 0.05 . De lo anterior se desprende que los estudiantes mejoraron la habilidad para detectar los conocimientos sobre la lectura realizada. En el momento de resolver el post-test fue fácil para los estudiantes detectar los conocimientos sobre el texto. Se confirmó lo que se expone en el modelo interactivo de Galvis, Castillo y Morales (2007): el lector, el texto y el contexto se interrelacionan, así el contexto es parte activa, es decir, el contexto influye en el proceso de la comprensión de la lectura.

En cuanto a la estrategia detección de dificultades en la comprensión, se notó un leve aumento del 54\% en el pre-test frente al 58\% en el post-test, porcentajes significativos al 0.05 y con un valor $t$-student significativo, lo que corrobora la efectividad de la estrategia en el mejoramiento de la comprensión del texto. Ríos (1991) involucra esta estrategia en la fase de supervisión con la pregunta ¿cómo determina cuáles son las partes del texto más difíciles de comprender?

Respecto de la estrategia conocimiento de las causas de las dificultades, el estudio mostró que en el pre-test los estudiantes obtuvieron un 53\%, que comparado con el pos-test (55\%) no es significativo, lo que indica que en el pre-test saben que tienen dificultades y las atribuyen a algo; en el post-test son conscientes de que al enfrentar cualquier lectura deben identificar las causas de las dificultades y probablemente al reconocerlas buscarán las posibles soluciones; según Ríos (1991), esto se da porque implica la siguiente pregunta: ¿por qué cree que se le dificulta la comprensión de esas partes del texto?, es decir, no solo cuáles son, sino las causas o razones de esas dificultades. En la primera parte, el estudiante no conoce las causas de sus dificultades, se aburre y abandona la lectura; mientras que en la segunda, se interesa por superar los obstáculos o limitaciones que puedan aparecer y sale adelante. Por otro lado, en la estrategia flexibilidad en el uso de estrategias, Ríos (1991) señala que comprende la pregunta: icuando se da cuenta 
de que no está comprendiendo, qué hace?, es decir, una vez identificadas las dificultades y sus causas, continúa con esta estrategia, para dar solución y actuar frente a ellas. El porcentaje en esta estrategia alcanzó un importante aumento del 39\%, pasando de $47 \%$ en el pre-test al $86 \%$ en el post-test, cifras significativas al 0.05 que verifican la eficacia de la intervención en el aprendizaje de esta estrategia, es decir, los estudiantes poseen una variedad de estrategias de solución, las ejecutan y autorregulan. Dentro de ese repertorio podrían nombrarse las siguientes: identificar mis conocimientos previos, consultar libros, profesores u otros medios, determinar el significado de las palabras utilizando el contexto de la lectura, parar cuando haya dudas y releer para clarificar, entre otras.

Finalmente, la última fase que propone Ríos (1991) es la evaluación, que comprende, como estrategia, la evaluación de la efectividad de las estrategias usadas: el porcentaje inicial de $55 \%$ se incrementó a $82 \%$, es decir, se observó una diferencia del $27 \%$ en el desempeño de los estudiantes. Considerando el valor $t$-student significativo; así, se confirma que la evaluación influye en la comprensión del texto, entendida con la pregunta: ¿qué pasos lleva a cabo durante la lectura para facilitar la comprensión del texto? De la misma manera, Galvis, Castillo y Morales (2007) proponen la evaluación como última estrategia que implica elaborar resúmenes y esquemas que den cuenta del texto, lo mismo que analizar errores cometidos y planear soluciones. Igualmente, en la intervención, para la fase de la evaluación, se instruyó a los estudiantes en la elaboración de mapas mentales para hacer asociación y síntesis de la información. Esta es una de las técnicas más efectivas en la organización y memorización de la información.

Por otro lado, en cuanto a la comprensión de texto, se estudiaron las siguientes categorías: vocabulario, detalles, superestructura y macroestructura de texto, identificación de la idea principal e inferencias.

Una vez realizado el análisis estadístico que compara los resultados obtenidos en el pre-test en relación con el post-test, se encontró el 95\% de confianza en el valor T-student, pues comparado con el valor crítico es significativo para la hipótesis, es decir esta se verificó, puesto que los resultados evidenciaron que al aplicar estrategias de comprensión lectora basadas en la metacognición para textos expositivos Sí se mejora significativamente la comprensión de ese tipo de textos.

Así, en la comprensión de vocabulario se notó un aumento del 52\% (pre-test) al $73 \%$ (post-test). Este incremento se dio porque los estudiantes incluyeron en su proceso lector el uso de redes de vocabulario; sinónimos; antónimos y la aplicación de la estrategia relacionada con conocimientos previos, que resulta efectiva, dado que el texto expositivo se 
caracteriza porque el vocabulario es específico y requiere del conocimiento y dominio del mismo.

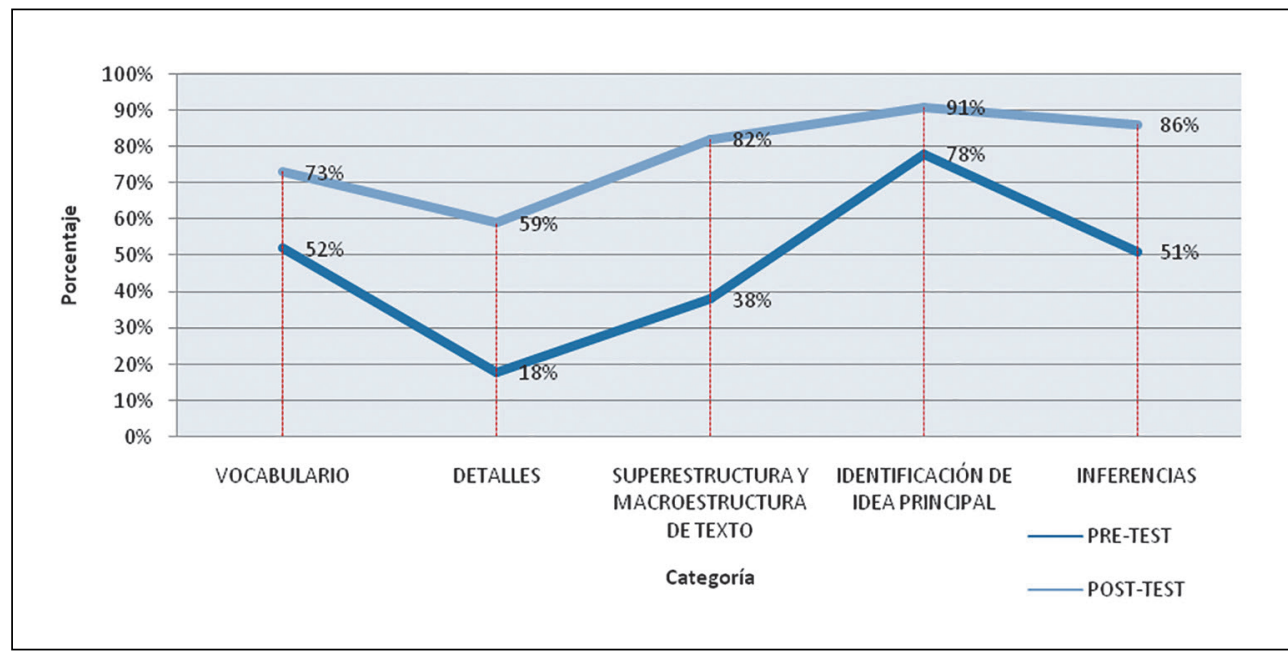

Figura 2. Porcentajes de respuestas correctas en el área de comprensión en el PRE-TEST Y POST-TEST

En la comprensión de detalles pasó del 18\% (pre-test) al 59\% (post-test). La diferencia corresponde al $41 \%$ que realmente es alto. La identificación de detalles sustentan y aclaran la idea principal, estos conforman la esencia del texto expositivo - explicativo, ya que los detalles relevantes especifican un tema en particular, permiten establecer jerarquías, transmitir datos, exponer y explicar información o conocimientos diversos. Las formas supralingüísticas abundan en este tipo de textos; en nuestro caso se utilizaron fotografías, ilustraciones, mapas, cuadros y marcas tipográficas que contribuyen a estructurar el contenido (Álvarez, 1996).

En la comprensión de superestructura y macroestructura de texto, pasó del 38\% (pre-test) al 82\% (post-test), es decir, también es significativamente alta. Ello se explica porque el estudiante aprendió a identificar el tema, las ideas fundamentales, la relación entre las ideas fundamentales, la información textual; es decir que, en los talleres realizados, se reforzaron los conocimientos previos, lo que favoreció la interrelación de estos últimos con la información textual en el post-test; además, la estrategia favoreció la construcción de imágenes del contenido textual en forma secuencial y organizada; en el caso del texto experimental, los estudiantes organizaron la secuencia de la lectura, valiéndose de imágenes mentales reforzadas con un video relacionado con la secuencia de la lectura. 
Por consiguiente, los estudiantes adquirieron una mejor comprensión de la coherencia y la cohesión textuales, y aprendieron a organizar de forma esquemática el significado global del texto.

En la comprensión para la identificación de la idea principal, se observó un incremento del 78\% (pre-test) al 91\%(post-test); con una diferencia del 13\%, y en la comprensión de inferencias pasó del 51\% (pre-test) al 86\% (post-test), con una diferencia del $35 \%$.

En cuanto al análisis de la comprensión de la idea principal y las inferencias (léxicas, de causalidad, de comparación, de especificación, de inclusión e inferencias macroestructurales) propuestas por Viramonte (2000), los resultados significativos provienen de la aplicación de la estrategia de conocimientos previos, dado que se requiere una información explícita que está dada por el texto y la implícita, o la que el lector infiere. Otra estrategia que influye es la identificación de la estructura del texto. Hacer preguntas inferenciales amplía y guía la comprensión, promueve el pensamiento crítico y centra al lector en la idea principal.

En síntesis, el desarrollo de los talleres y la enseñanza de las estrategias metacognitivas demostraron que todas ellas aplicadas en las fases de planificación, supervisión y evaluación influyen en la elaboración de inferencias y en la confirmación o modificación de estas, de acuerdo con las experiencias previas y lo explícito en el texto, como lo afirman Mendoza y Briz (2003), aspecto que está presente en todo el proceso de la lectura. Por otra parte, según los resultados, los estudiantes mostraron una mayor habilidad para elaborar inferencias del texto.

\section{Conclusiones}

La presente investigación llegó a las siguientes conclusiones: se mejoró significativamente la comprensión del texto expositivo-explicativo mediante la aplicación de estrategias metacognitivas en la planificación, supervisión y evaluación de la lectura, es decir, en las fases que se desarrolló la lectura.

La aplicación de las estrategias metacognitivas a través de la implementación de talleres, favoreció la activación de procesos conscientes y no automáticos; en esta medida, los estudiantes reconocieron su propio proceso y la planificación del mismo, involucraron procesos como la atención, la percepción y la memoria. Los estudiantes mostraron mayor habilidad para la autorregulación y el control en su propio proceso de la lectura de forma consciente, esto implica el cuestionamiento y la detección de las fases y estrategias empleadas durante la lectura. Por lo tanto, consideramos que la práctica constante de estas 
estrategias favorece en el estudiante la agudeza y la habilidad en la aplicación de estrategias metacognitivas y su efectividad en el proceso lector. Lo anterior incide en la satisfacción personal, en el autoconocimiento y, principalmente, en la motivación hacia la lectura.

Los resultados de esta investigación aportan formación pedagógica a los maestros, ya que si ellos reciben formación en la enseñanza y aprendizaje de las estrategias metacognitivas para mejorar la comprensión de lectura y la capacidad de deducción e inferencias en los estudiantes, ello se verá reflejado en su práctica pedagógica y, por ende, en la mejora de la calidad educativa.

Otro aporte de esta investigación a los docentes consiste en la importancia de identificar el tipo de texto que se lee en las aulas, ya que se debe desarrollar en los estudiantes habilidades específicas para cada una de las tipologías de los textos; sin el ánimo de demeritar los otros tipos de textos, el expositivo- explicativo aporta conocimientos y transmite saberes, información e ideas nuevas.

En síntesis, la prioridad de la investigación fue desarrollar en los estudiantes sus conocimientos previos; el conocimiento o teoría de mundo; la detección de pistas que ofrece el texto; la identificación de la estructura y cohesión del texto; la capacidad de realizar inferencias durante y después de la lectura, lo cual consolida la comprensión lectora, dado que ello implica la habilidad para establecer las siguientes relaciones, entre otras: causa - efecto, pregunta - respuesta, clasificaciones - colección, definición - descripción, síntesis - análisis, y para identificar en el texto marcadores discursivos (conectores lógicos y organizadores textuales).

\section{Referencias bibliográficas}

Álvarez Angulo, T. (1996). El texto expositivo-explicativo: su superestructura y características textuales. Didáctica, Lenguaje Literatura, (8), 29. doi: 10.5209/rev_DIDA.1996.v8.20824.

Arrieta Arvilla, L.M. (2013). La construcción de los sujetos en el discurso del expresidente Uribe Vélez: un análisis desde las tonalidades valorativas y los actos de habla. Cuadernos de Lingüística Hispánica, (21), 103-116. doi: http://dx.doi.org/10.19053/0121053X.1952

Brown, A. L., Armbruster, B.B., \& Baker, L. (1986). The role of metacognition in reading and studying. En J. Orasanu (Ed.). Reading comprehension: From research to practice. Hillsdale, NJ: L.E.A. 
Chirilos, N.M. (2014). Estrategias metacognitivas en el proceso de investigación científica. (Tesis de Doctorado). Universidad de Córdoba, Venezuela. Recuperado de https://www.google.com. co $/$ webhp? sourceid $=$ chrome-instant\&ion $=1 \&$ espv $=2 \&$ ie $=U T F-8 \# q=2014000000861$

Cooper, J.D. (1998). Cómo mejorar la comprensión lectora. Madrid: Visor Fotocomposición. UPEL. Maracay. Universidad Pedagógica Experimental Libertador. Núcleo Biodiversidad.

Flavell, J.H. (1979). Metacognition and cognitive monitoring: A new area of cognitive developmental inquiry. American Psychologist.

Flavell, J., \& Wellman, H. (1977). Metamemory. En R. Kail y J. Hagen (Eds.). Perspectives on the development of memory and cognition. Hillsdale: LEA.

Galvis, A., Castillo, M., \& Morales, D. (2007). Estrategias y enseñanza-aprendizaje de la lectura. Folios, 2(26), 27-38.

Heit, I. A. (2011). Estrategias metacognitivas de comprensión lectora y eficacia en la Asignatura Lengua y Literatura [en línea]. Tesis de Licenciatura, Universidad Católica Argentina, Facultad Teresa de Ávila. Departamento de Humanidades. Recuperado de http://www.fisica. $\mathrm{ru} / \mathrm{dfmg} /$ teacher/archivos/ESTRATEGIAS-METACOGNITIVAS-OSCAR-M-TRONCOSO.pdf

Lomas, C. (1999). Cómo enseñar a hacer cosas con las palabras. Teoría y práctica de la educación lingüística. Barcelona: Paidós.

Mendoza Fillola, A., \& Briz Villanueva, E. (2003). Didáctica de la Lengua y la Literatura para la Primaria. Madrid: Pearson Educación.

Nieto Ruiz, L.F. (2011). El papel del lenguaje en las estrategias discursivas para fomentar la enseñanza-aprendizaje. Cuadernos de Lingüística Hispánica, (18), 135-153. Recuperado de http://revistas.uptc.edu.co/revistas/index.php/ linguistica_hispanica/article/view/438.

Ríos Cabrera, P. (1991). Metacognición y comprensión de la lectura. En A. Puente (Comp.). Comprensión de la lectura y acción docente. Madrid: Pirámide.

Patiño Vélez, J. P., \& Restrepo Sánchez, P. A. (2013). Estrategias metacognitivas: Herramientas para mejorar la comprensión textual en estudiantes de básica primaria. Recuperado de: http://200.24.17.68:8080/jspui/handle/123456789/1573

Solé, I. (1994). Estrategias de lectura. Colección MIE. Barcelona: Graó.

Santiago Galvis, Á. W., Castillo, M.C., \& Vega R. J. (2005). Lectura, metacognición y evaluación. Bogotá: Alejandría Libros.

Sanz, M. (2010). Competencias cognitivas en Educación Superior. Madrid: Narcea. 
Tardif, J. (1997). La evaluación del saber- leer: un asunto más de competencia que de actuación. En E. Rodríguez \& E. Lager (Coords.). La lectura. Cali: Universidad del Valle.

Torres Cruz, D.L., \& Hurtado, L.M. (2016). Mecanismos de control que determinan la identidad a través del lenguaje en un contexto escolar: un estudio para la interacción social. Cuadernos de Lingüística Hispánica, (28), 19-40. doi: http://dx.doi.org/10.19053/0121053X.4906

Vásquez Cantillo, A. (2012). Modelos pedagógicos: medios, no fines de la educación. Cuadernos de Lingüística Hispánica, (19), 157-168. Recuperado de http://revistas.uptc.edu.co/revistas/ index.php/linguistica_hispanica/article/view/457/457

Viramonte, M. (2000). Comprensión Lectora. Dificultades estratégicas en resolución de preguntas inferenciales. Buenos Aires: Colihue. 